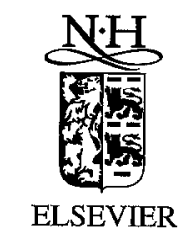

Surface Science 377-379 (1997) 687-690

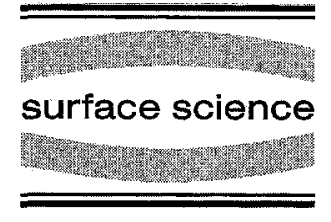

\title{
Non-thermal kinetic-energy distributions of desorbed Na dimers
}

\author{
J. Viereck ${ }^{a}$, M. Stuke ${ }^{b}$, F. Träger ${ }^{a, *}$ \\ ${ }^{a}$ Fachbereich Physik, Universität Kassel, Heinrich-Plett-Strasse 40, D-34132 Kassel, Germany \\ b Max-Planck-Institut fïr biophysikalische Chemie, P.O. Box 2841, D-37018 Göttingen, Germany \\ Received 1 August 1996; accepted for publication 15 October 1996
}

\begin{abstract}
Experiments are reported which demonstrate that $\mathrm{Na}$ dimers can be desorbed in a non-thermal reaction if rough Na surfaces are irradiated with pulsed laser light of $\lambda=355 \mathrm{~nm}$ at low laser fluences. This follows from the kinetic-energy distributions of the dimers, which were determined by time-of-flight measurements using a second laser at $\lambda=248 \mathrm{~nm}$ for photoionization. The rate of desorbed dimers can even surmount the rate of detached atoms. Obviously, the dimers constitute highly stable sub-units of the surface which can be released without breaking into individual atoms.
\end{abstract}

Keywords: Alkali metals; Clusters; Photon stimulated desorption (PSD)

\section{Introduction}

Whereas laser-induced desorption of molecules has been studied in great detail in the past, only a few studies dealt with laser-stimulated desorption of metal or semiconductor aggregates which contain two or more atoms [1-4]. For example, Brewer et al. [1] studied nanosecond and femtosecond UV laser ablation of CdTe (100) surfaces. They used angularly resolved time-of-flight measurements to probe the velocity and angular distributions of desorbing $\mathrm{Cd}$ atoms as well as $\mathrm{Te}_{2}$ molecules, and found that the time-of-flight distributions of both ejected products could be described by Maxwellians. Götz et al. [3] investigated the mechanisms of laser-induced desorption of $\mathrm{Na}$ atoms and $\mathrm{Na}$ dimers from rough $\mathrm{Na}$ surfaces. The results showed that for a laser wavelength of $\lambda=$

\footnotetext{
* Corresponding author. Fax: +49 5618044518 ; e-mail: traeger@physik.uni-kassel.de
}

$355 \mathrm{~nm}$ and fluences above $40 \mathrm{~mJ} \mathrm{~cm} \mathrm{~cm}^{-2}$, desorption of $\mathrm{Na}$ dimers takes place. With increasing laser fluence, the fraction of detached $\mathrm{Na}$ dimers as compared to $\mathrm{Na}$ atoms increases. The experimentally observed energy distributions suggested that the dimers were directly desorbed from the surface in a thermal process, and not formed by gas-phase collisions of desorbed atoms [3]. As a continuation of this work, the present paper reports studies of $\mathrm{Na}$ dimer desorption at low laser fluences. The objective was first to clarify in these and in future experiments under which conditions clusters $\mathrm{X}_{n}$ (with $n \geq 2$ ) can be desorbed with laser light in a non-thermal process, and secondly to elucidate the underlying mechanism.

To stimulate desorption with laser radiation, electronic excitation at the surface has to be accomplished as the first step. It is usually followed by rapid relaxation and generation of heat causing thermal bond-breaking. Under certain conditions, however, the electron-phonon coupling can be 
reduced in strength, making non-thermal desorption possible (see, e.g., Ref. [5]) of atomic species [6-9] as a direct result of repulsive electronic excitation. Further, it turns out that desorption along these lines takes place preferentially from defects of the surface, i.e. from sites with particularly low coordination numbers $[5,10]$. Therefore, in order to study the desorption of clusters, it seems desirable to use surfaces with the largest possible number of such sites. For this purpose, surfaces with large roughness have been prepared here by the deposition of metal atoms on dielectric substrates held at low temperature. As in earlier work on atom desorption [11], sodium adsorbed on quartz served as a model system. Initially, the deposited atoms form small particles with the substrate defects acting as nucleation centers [12]. If deposition is continued, the clusters finally grow together, generating a film.

\section{Experimental}

The experimental arrangement has been described in detail elsewhere [3] and basically consists of an ultrahigh vacuum system with the sample, two lasers for stimulating desorption and photoionizing the desorption products, and a time-of-flight mass spectrometer. A thermal atomic beam of $\mathrm{Na}$ atoms with a well-defined constant flux is directed onto the quartz substrate in order to deposit a predetcrmined coverage of atoms onto the surface held at $80 \mathrm{~K}$. The experiments reported here were carried out at low coverage, and in addition at low laser fluence to keep the desorption rate small, and to avoid gas-phase collisions of the desorbed species and thus measure their genuine kinetic-energy distribution. After preparation the sample was irradiated with the light of a Nd:YAG laser at $\lambda=355 \mathrm{~nm}$ in order to stimulate desorption. The angle of incidence was $50^{\circ}$ with respect to the surface normal, and the pulse duration was about $7 \mathrm{~ns}$ at a repetition rate of $10 \mathrm{~Hz}$. At a distance of $21 \mathrm{~mm}$ in front of the substrate, the species detached from the cluster surface are ionized with the light of an excimer laser operating at $\lambda=248 \mathrm{~nm}$. The corresponding photon energy lies only a little above the ionization threshold of $\mathrm{Na}_{2}$. Therefore, if compared to earlier work with $\lambda=193 \mathrm{~nm}$, where strong fragmentation of the Na-dimers was observed [3], "soft" ionization of the desorbed dimers is accomplished and there is only very little dissociation into atoms. The generated ions pass the time-of-flight mass spectrometer and are finally detected with a secondary electron multiplier. The ion signal is processed with a boxcar integrator and stored in a computer. By varying the delay time between the two laser pulses used for desorption and ionization, the time-of-flight distributions of the desorbed $\mathrm{Na}$-dimers were determined and then converted into flux distributions as a function of translational energy [13].

\section{Results and discussion}

As an example, Fig. 1 shows kinetic-energy distributions obtained by desorbing dimers with laser light of $\lambda=355 \mathrm{~nm}$ and a total coverage of $7.2 \times 10^{14}$ atoms cm$~^{-2}$, corresponding to an average particle size of $R_{\text {ave }}=7 \mathrm{~nm}$. The fluence of the $\mathrm{Nd}$ :YAG laser was increased first from $\Phi=$ $3 \mathrm{~mJ} \mathrm{~cm}^{-2}$ (Fig. 1a) to a value of $\Phi=5 \mathrm{~mJ} \mathrm{~cm}^{-2}$ (Fig. 1b). In both cases the kinetic-energy distribution shows a maximum. In contrast, the distributions change drastically at larger fluences of, for example, $\Phi=9 \mathrm{~mJ} \mathrm{~cm}^{-2}$ (Fig. 1c). It no longer exhibits a maximum, but falls off monotonously, with dimers of very low kinetic energy contributing substantially to the signal. This distribution can be fitted by an exponential (const. $\exp \left(-E_{\text {kin }} / k T\right)$ ), represented by the dashed line in Fig. 1c), indicating that thermal bond-breaking is responsible for desorption. Laser-induced thermal desorption of $\mathrm{Na}$ dimers is also observed if sodium surfaces are irradiated by light of $\lambda=532 \mathrm{~nm}$ [14].

In further measurements the total coverage and the laser fluence were varied systematically. At low laser fluences, a maximum is observed for all kineticenergy distributions of the desorbed dimers. The data are compiled in Fig. 2, which shows the positions of the maxima of the distributions for five values of the laser fluence and four different coverages. The most essential result is that the positions of these maxima at $E_{\mathrm{kin}}=0.06(1) \mathrm{eV}$ are identical within the cxpcrimental crror of $0.01 \mathrm{cV}$, and depend neither on particle size nor on laser fluence. 

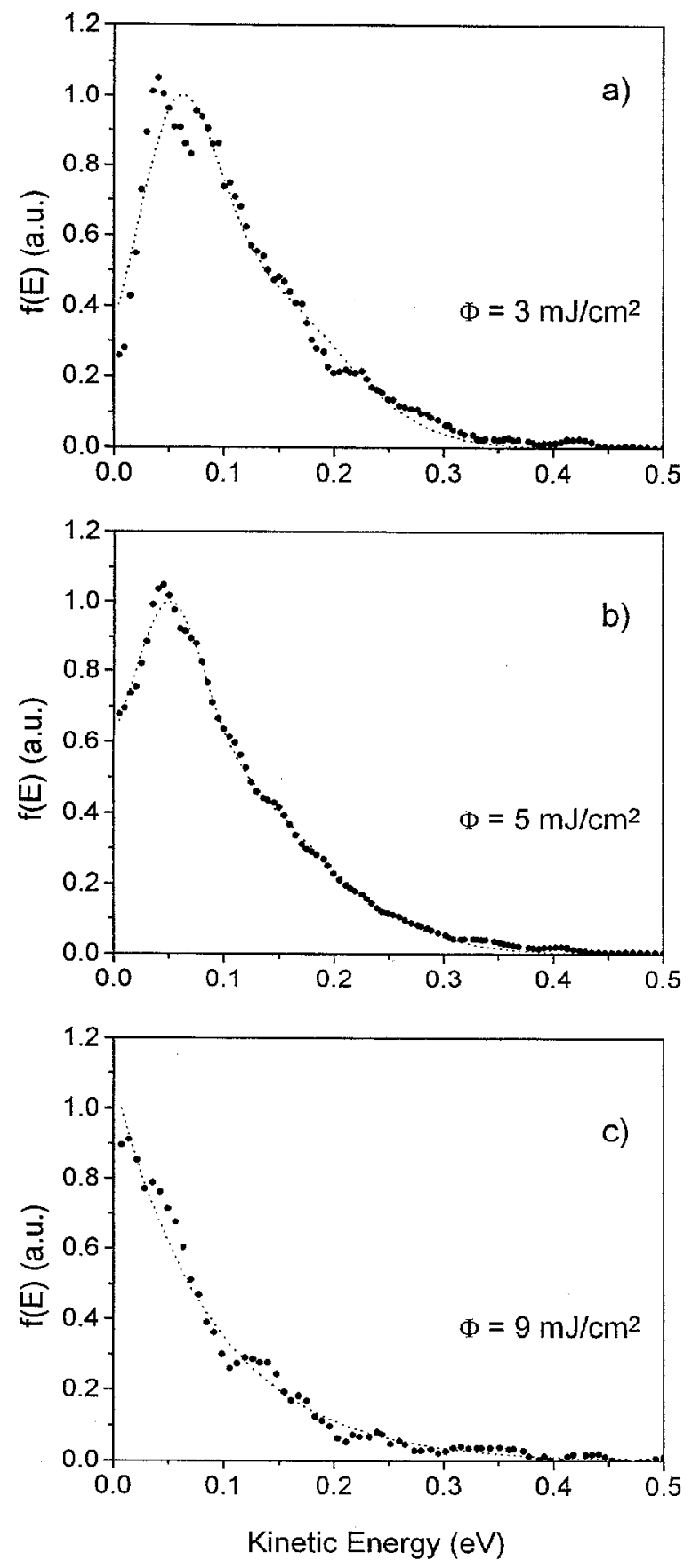

Fig. 1. Kinetic-energy distributions normalized to the maximum rate of $\mathrm{Na}$ dimers desorbed at $\lambda=355 \mathrm{~nm}$ and a total coverage of $7.2 \times 10^{14}$ atoms cm $\mathrm{cm}^{-2}\left(R_{\text {ave }}=7 \mathrm{~nm}\right)$ with fluences of (a) 3 , (b) 5 and (c) $9 \mathrm{~mJ} \mathrm{~cm}{ }^{-2}$. The wavelength of the ionization laser was set to $\lambda=248 \mathrm{~nm}$.

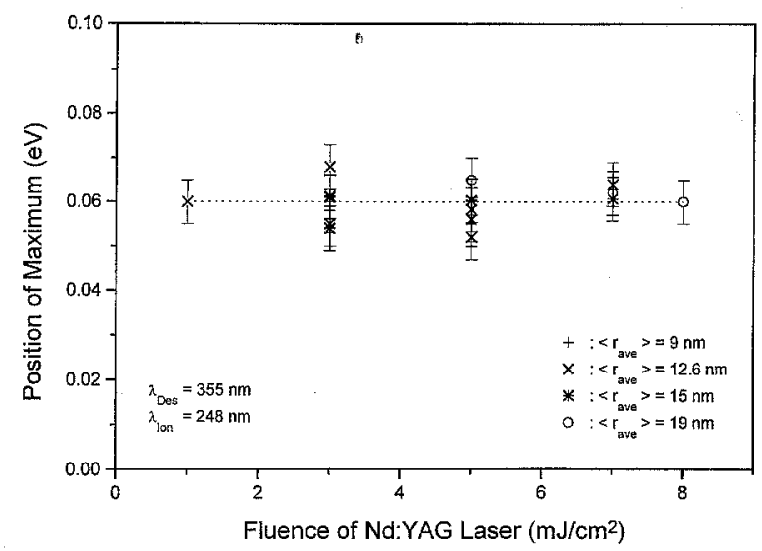

Fig. 2. Positions of the maxima of the kinetıc-energy distributions of $\mathrm{Na}$ dimers desorbed with laser light of $\lambda=355 \mathrm{~nm}$. The laser fluence was varied between 1 and $8 \mathrm{~mJ} \mathrm{~cm}^{-2}$, and the average particle size ranged from 7 to $15 \mathrm{~nm}$.

Fig. 3 shows the integral desorption rate obtained with $\lambda=355 \mathrm{~nm}$ as a function of laser fluence for the fixed coverage of $3.6 \times 10^{15}$ atoms $\mathrm{cm}^{-2}\left(R_{\text {ave }}=\right.$ $15 \mathrm{~nm}$ ). At low values, the integral desorption rate is linearly dependent on the laser fluence. Above approximately $\Phi=7 \pm 1 \mathrm{~mJ} \mathrm{~cm}{ }^{-2}$, however, it grows exponentially. At the same time, the shape of the kinetic-energy distribution changes from a curve exhibiting a maximum to an exponential decrease (see Figs. $1 b$ and 1c).

We note that desorption of sodium dimers $\left(\mathrm{Na}_{2}\right)$

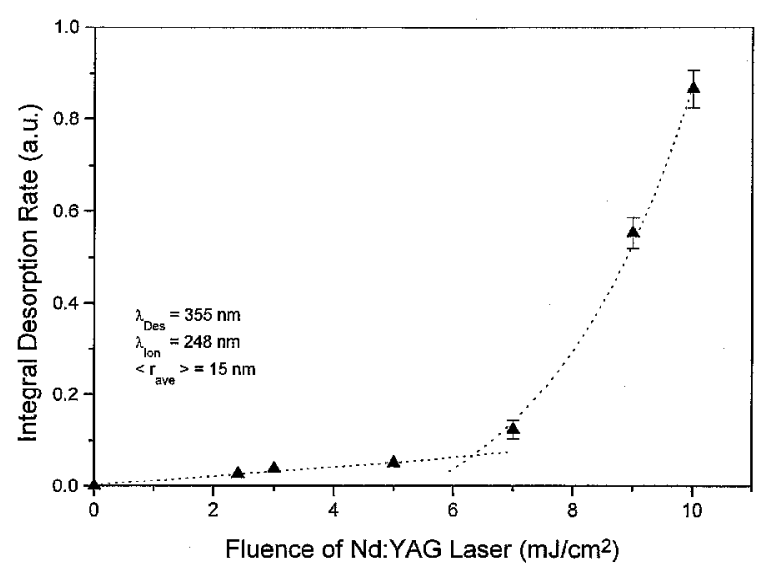

Fig. 3. Integral desorption rate of $\mathrm{Na}$ dimers desorbed with a laser wavelength of $\lambda=355 \mathrm{~nm}$ as a function of laser fluence. The coverage was $3.6 \times 10^{-15}$ atoms $\mathrm{cm}^{2}\left(R_{\mathrm{ave}}=12 \mathrm{~nm}\right)$. The fluence was varied between 1 and $10 \mathrm{~mJ} \mathrm{~cm}^{-2}$. 
is readily detectable for small laser fluences. At fluences lower than $\Phi=7 \pm 1 \mathrm{~mJ} \mathrm{~cm}{ }^{-2}$, it obviously occurs as a non-thermal reaction. This is supported by the shape of the kinetic-energy distributions of the detcetcd dimcrs, the linear fluence dependence and by the constant value of the most likely kinetic energy of $E_{\text {kin }}=0.06(1) \mathrm{eV}$. This value turns out to be independent of coverage (i.e. particle size), and, as mentioned above, of the light intensity. Furthermore, the $\mathrm{Na}$ particles only experience a moderate temperature rise under the conditions quoted above [5]. Also, the transition from nonthermal to thermal desorption as a function of incrcasing laser fluence can be identified. It is reflected in the appearance of exponential kineticenergy distributions which gradually replace the curves exhibiting a maximum (Fig. 1), and in the simultaneous transition from a linear to an exponential dependence of the integral desorption rate (Fig. 3). This agrees very well with the results of earlier work, which showed thermal desorption of sodium dimers for high laser fluences [3].

The results reported here indicate that the dimers are desorbed directly from the surface, and are not formed by gas-phase collisions of the desorbing atoms. The following two arguments support this conclusion. In order to form the dimers by gasphase collisions in front of the surface, three-body collisions would be required. They occur, however, with sufficient probability only at a large sodium vapour pressure which cannot be produced by the low laser fluences used in this experiment. In addition, three-body collisions would cause pronounced thermalization of the kinetic-energy distributions of the sodium dimers and atoms [15], in strong contrast to the observed energy distributions of the $\mathrm{Na}$ dimers reported in the present paper and of $\mathrm{Na}$ atoms published elsewhere [11].

The large ratio of the desorption rates of dimers and atoms (3-4) indicates that the dimers obviously constitute highly stable sub-units of the metal surface which can be released in a photochemical reaction without breaking into individual atoms. This seems to imply that the dimers are oriented on the surface in such a way that the axis connecting the two $\mathrm{Na}$ atoms is not parallel to the substrate surface, but rather points in the direction of the surface normal. The repulsive electronic excitation preceeding desorption must then be localized in the region between the dimer and the remaining substrate atoms.

In future experiments, the non-thermal reaclion as well as the interplay between thermal and nonthermal bond-breaking will be studied in more detail. For example, the angular distribution of the desorbed atoms and dimers should give further information on the desorption mechanism. A very interesting question is also whether or not desorption of aggregates $\mathrm{X}_{n}$ with $n>2$ can be stimulated. In order to address this problem, use of longer ionization wavelengths is essential to prevent photofragmentation of the larger aggregates [16].

\section{Acknowledgements}

Financial support of the Fond der Chemischen Industrie (F.T.) and BMBF 13N 61597 is gratefully acknowledged.

\section{References}

[1] P.D. Brewer, M. Späth and M. Stuke, Mater. Res. Soc. Symp. Proc. 334 (1994) 245.

[2] D.L. Pappas, K.L. Saenger, J. Cuomo and R.W. Dreyfus, J. Appl. Phys. 72 (1992) 3966.

[3] T. Götz, M. Bergt, W. Hoheisel, F. Träger and M. Stuke, Appl. Surf. Sci. 96-98 (1996) 280.

[4] A. Drescher, J. Kitching, J.E. Crawford, J.K.P. Lee and G. Thekkadath, Z. Phys. D 19 (1991) 203.

[5] W. Hoheisel, M. Vollmer and F. Träger, Phys. Rev. B 48 (1993) 17463.

[6] W. Hoheisel, K. Jungmann, M. Vollmer, R. Weidenauer and F. Träger, Phys. Rev. Lett. 60 (1988) 1649.

[7] A.M. Bonch-Brucvich, T.A. Vartanyan, Yu.N. Maksimov, S.G. Przhibel'skii and V.V. Khromov, Surf. Sci. 307-309 (1994) 350.

[8] H.S. Kim and H. Helvajian, J. Chem. Phys. 95 (1991) 6623.

[9] M.J. Shea and R.N. Compton, Phys. Rev. B 47 (1993) 9967.

[10] K. Ishikawa, J. Kawasaki, Y. Nakai and M. Itoh, Surf. Sci. 349 (1996) L152.

[11] T. Götz, M. Bergt, W. Hoheisel, F. Träger and M. Stuke, Appl. Phys. A 63 (1996) 315.

[12] J.A. Venables, Surf. Sci. 299/300 (1994) 798.

[13] T.D. Bennett, D.J. Krainovich and C.P. Grigoropoulos, Phys. Rev. Lett. 76 (1995) 1659.

[14] J. Viereck, M. Stuke and F. Träger, Appl. Phys. A 64 (1997) 149.

[15] D. Sibold and H.M. Urbassek, Phys. Rev. A 43 (1991) 6722.

[16」 K.J. Peterson, P.D. Dao, R.W. Farley and A.W. Castleman, J. Chem. Phys. 80 (1984) 1780. 